\title{
LETTERS
}

\section{Making treatment recommendations during the COVID-19 pandemic}

In their article on pandemic palliative care, Arya and colleagues offer suggested language for discussing a treatment plan with patients who are unlikely to survive a critical illness, but whose current treatment plan would include life-sustaining therapies, if indicated. ${ }^{1}$ I would like to highlight the important skill of making treatment recommendations. Patients and families facing serious illness often feel anxiety in the face of uncertainty. During a pandemic, this anxiety is amplified as patients and families may be physically separated, and guidance from government and institutions may change rapidly. Our ability as clinicians to respond empathically, share prognostic concerns, listen to what matters most, and use this information to make treatment recommendations aligned to the goals of patients and families can garner trust and alleviate distress.

Training programs on communication skills are available to assist clinicians in navigating goals-of-care discussions and making treatment recommendations. These programs, which use structured guides and teach empathic communication skills, are associated with favourable patient outcomes. ${ }^{2,3}$ Importantly, these guides move clinicians away from asking patients what they want (e.g., "Would you want treatment $[\mathrm{x}$ ] if you got sicker?"), to recommending treatments aligned to patient and family values (e.g., "Based on what I hear you saying, I'd like to make a recommendation that would support you to achieve your goals of [a] and [b]. Is that okay?"). In my experience as a palliative care physician, patients and families expect clinicians to make values-based recommendations and are comforted by not feeling burdened to make "the right decision" themselves.

\section{Warren Lewin MD}

Palliative care physician, University Health Network, Toronto Western

Hospital, Toronto, Ont.

Cite as: CMAJ 2020 May 11;192:E521. doi: 10.1503/cmaj.75485

\section{References}

1. Arya A, Buchman S, Gagnon B, et al. Pandemic palliative care: beyond ventilators and saving lives. CMAJ 2020;192:E400-4.

2. Childers JW, Back AL, Tulsky JA, et al. REMAP: a framework for goals of care conversations. J Oncol Pract 2017;13:e844-50.

3. Bernacki R, Hutchings M, Vick J, et al. Development of the Serious Illness Care Program: a randomised controlled trial of a palliative care communication intervention. BMJ Open 2015;5:e009032.

Competing interests: None declared. 